\title{
Radionuclide Lymphoscintigraphy in the Diagnosis of Idiopathic Congenital Lymphedema- a Case Report
}

\author{
Shamim M F Begum, Nasreen Sultana, Rahima Parveen, Amardeep Chaudhury and Fatima Begum \\ National Institute of Nuclear Medicine and Allied Sciences (NINMAS), Dhaka, Bangladesh \\ Correspondence Address : Prof. Dr. Shamim Momtaz Ferdousi Begum, Professor and Head Nuclear Nephrology Division, National \\ Institute of Nuclear Medicine \& Allied Sciences, BAEC, Email: pragyna06@yahoo.com
}

\begin{abstract}
Congenital lymphedema is the rarest form of primary lymphedema, accounting for approximately 1: 60,000 live births. Congenital lymphedema can be classified into familial (hereditary) and idiopathic (non hereditary) subgroups. When congenital lymphedema is of the hereditary form the eponym Milroy disease is applied. Lymphedema without any dysmorphic features and no family history of lymphedema the eponym idiopathic congenital lymphedema is utilized. Etiology of idiopathic primary congenital lymphedema is unknown and a de novo genetic event of genes involved in lymphangiogenesis is a possibility. Radionuclide lymphoscintigraphy is considered as a gold standard for the diagnosis of lymphedema. This reported interesting case was an eleven months old girl having swelling of right lower limb since birth. There was no family history of lymphedema and no any dysmorphic features consistent with Milroy disease. The primary diagnosis was congenital lymphedema of idiopathic (non hereditary) subtype. Lymphoscintigraphic with Technetium-99m (Tc- 99m nanocolloid) revealed no lymphatic channels or inguinal lymph nodes on right side in early or delayed images up to 2 hours views and the diagnosis of primary idiopathic congenital lymphedema was confirmed.
\end{abstract}

Key words: Congenital lymphedema, lymphoscintigraphy

\section{INTRODUCTION}

Lymphedema is defined as regional accumulation of excessive interstitial protein-rich fluid caused primarily by an imbalance between the inflow and the removal of interstitial fluid and protein, and secondarily by a malformation or malfunction of the lymphatic System

(1). There is two major categories of lymphedema: primary and secondary (2). Primary lymphedema is a rare disease that results from impaired lymphatic drainage. It is estimated to occur in about 1.15 in 100,000 persons who are less than 20 years old with 1:3 male female ratios

(3). Causes of primary lymphedema are due to congenital absence, hypoplasia or under development of the superficial lymphatic channel. The disease is limited to the leg but arms, hand and feet can also be affected (4). If lymphatics are damaged or blocked by any pathologic process like infection, surgical excision, neoplasm, irradiation, or trauma, the interstitial fluid builds up in the tissues causing secondary lymphedema (5).

Three form of primary lymphedema have been described: lymphedema tarda, with onset in the late second or third decade of life (1); lymphedema praecox with onset at or near puberty (2); and congenital lymphedema, noticed at birth or soon thereafter $(3,4)$. Congenital lymphedema is the rarest form of primary lymphedema, accounting for approximately 1:60,000 live births (1). Congenital lymphedema can be classified into familial (hereditary) and idiopathic (non hereditary) subgroups. When lymphedema without any dysmorphic features and no family history of lymphedema the eponym idiopathic congenital lymphedema is utilized. Etiology of idiopathic primary congenital lymphedema is unknown and a de novo genetic event of genes involved in lymphangiogenesis is a possibility (4). Here we reported a case of idiopathic congenital lymphedema and the diagnosis was confirmed by Tc-99m nanocolloid lymphoscintigraphy.

\section{CASE REPORT}

A 11 months old girl was referred to National Institute of Nuclear Medicine \& Allied Sciences (NINMAS) with the advice of radionuclide lymphoscintigraphy from outpatient department of Dhaka Medical College Hospital. The patient was presented with swelling of right lower limb since birth. According to her mother's statement, the baby was born with a 'swollen' right leg. Initially the swelling was confined to the foot and gradually it was progressing with the involvement of right leg. The girl had the history of gradual swelling of hands on both sides. The growth and mile stone of development of the patient was normal. She was born at term by caesarian section and her mother reported no 
postnatal complications. She was the second child of nonconsanguineous parents. There were no any episodes of acute illness, no history of lymphangitis or cellulitis. There was no history of travel to areas endemic for conditions causing lymphoedema. Neither of the parents had same illnesses, and there was no history of other family members with a similar condition.

Physical examination revealed non-pitting, non tender edema of right foot, right leg and both hands (Figure1 and Figure 2). She had unilateral swelling of the face and mild peri-orbital edema. The edema was marked at the palm and dorsum of the hands and foot with sparing of the distal digits (Figure 1 and 2).

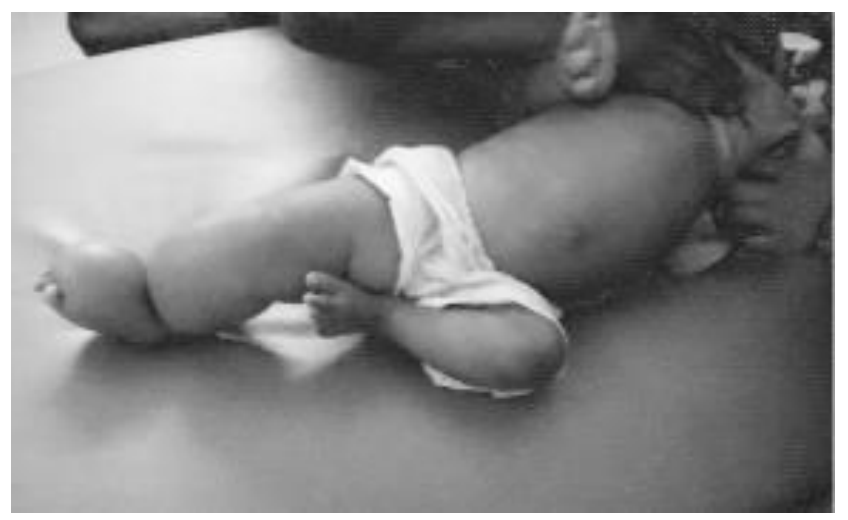

Figure 1: Swelling of right lower limb

There was no evidence of lymphadenopathy or cardiac failure. She had no other dermatological stigmata or any dysmorphic features. The circumferences in different regions on the right foot and leg were 2 to $6 \mathrm{~cm}$ greater than corresponding regions on the left (Figure 2).

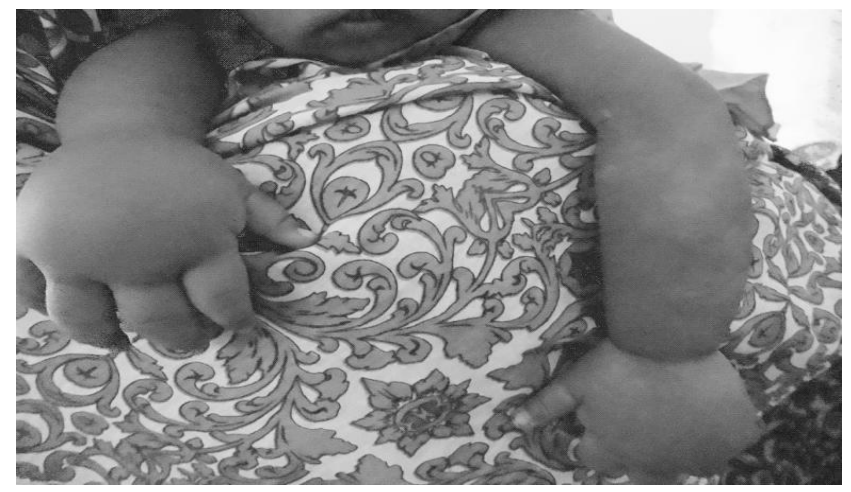

Figure 2: Swelling of both upper limbs

There was no localized overgrowth of bone and no varicose veins. Her nails were normal. Biochemical tests were done to exclude hepatic or renal etiologies and urine analysis to exclude proteinuria. All of these laboratory findings were normal. Doppler ultrasonography of affected limbs (Figure 3, 4) as well as pelvic and abdominal ultrasonography was done to exclude deep vein thrombosis and obstructive lesions that may present with unilateral limb swelling and was found normal.

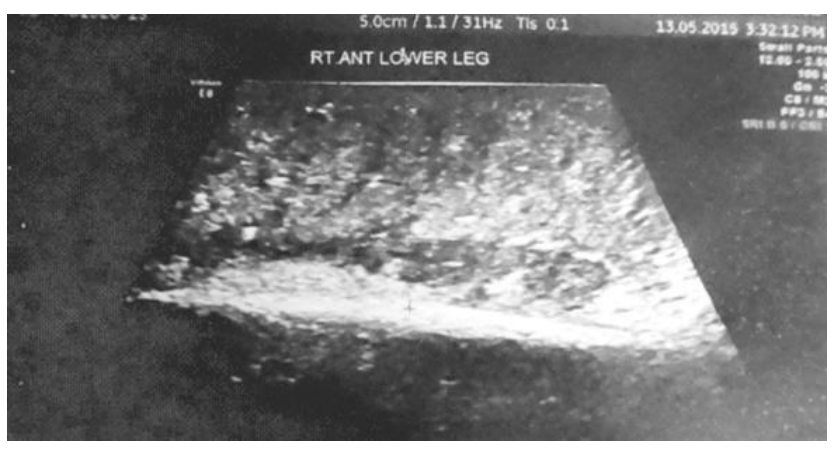

Figure 3: 2D Ultrasonogram of right leg shows grossly increased thickness of the dermis and increased thickness of subcutaneous layer of the right leg.

Complement fixation Test (CFT) for filariasis was negative. The primary diagnosis was congenital lymphoedema. The diagnosis of idiopathic primary congenital lymphedema was confirmed by doing Tc $-99 \mathrm{~m}$ nanocolloid lymphoscintigraphy. Lymphoscintigraphic findings revealed no lymphatic channels or inguinal lymphnodes on right side was visualized in early or delayed images up to 2 hours. However, on left side evidence of significant dilatation of lymphatic channels from inguinal lymph nodes (visualized within 20 minutes) and seems to be communicating with abdominal lymphatic channels (Figure 5 and 6). The patient was treated conservatively.

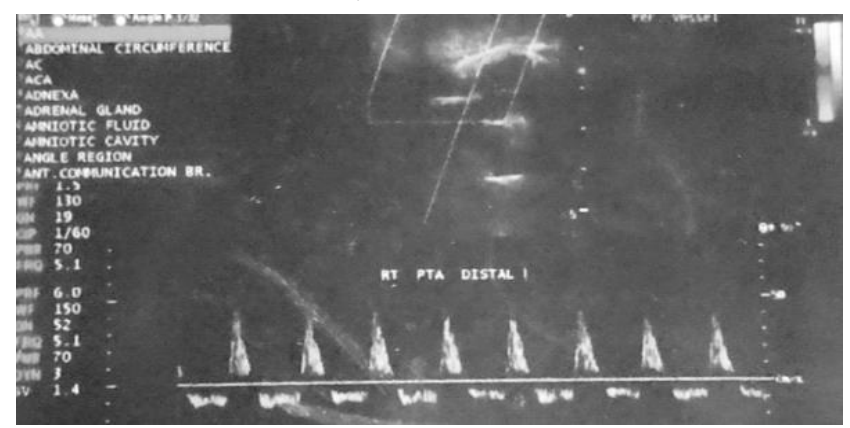

Figure 4: Normal duplex study of right lower limb vessels.

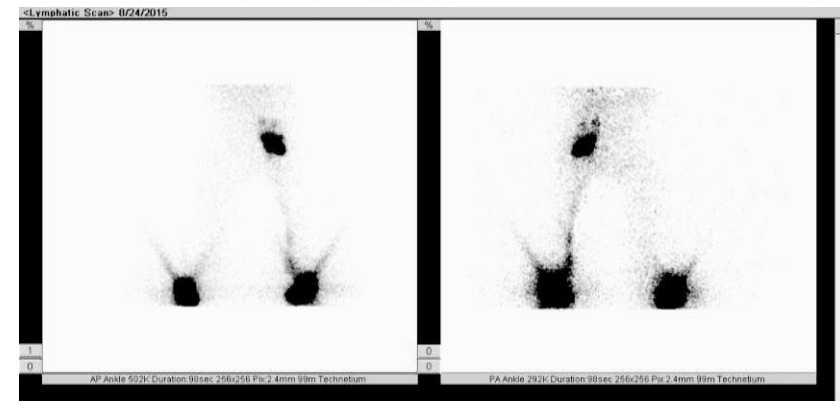

Figure 5: Early views of Tc $\mathbf{- 9 9 m}$ nanocolloid lymphoscintigraphy of lower limbs. 


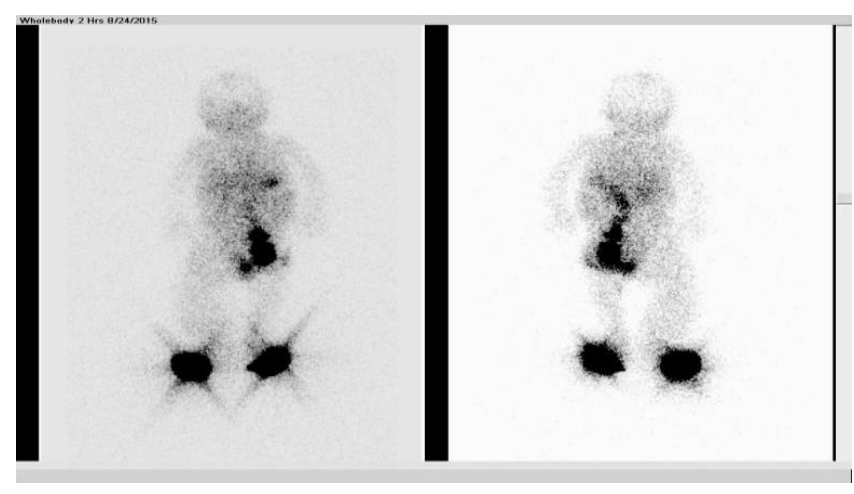

Figure 6 : Delayed views Tc-99m nanocolloid lymphoscintigraphy shows non-visualized lymphatic channel and lymph nodes in right lower limb with no ascent of tracer into the lymphatic vessels.

\section{DISCUSSION}

Idiopathic primary lymphedema is a rare disorder causing persistent swelling in an extremity due to impaired lymphatic drainage. Wananukul et al reported a case of idiopathic primary congenital lymphedema involving lower limbs and genitalia but patient had no family history of lymphedema and also had no dysmorphic feature (5). Sim et al also reported a case of lymphedema without any family history and dysmorphic features and he categorized the case as an idiopathic congenital lymphedema. Our patient also had no family history of lymphedema and no dysmorphic features. We diagnosed this case as idiopathic primary congenital lymphedema. Studies reported lower limb involvement constitutes most cases of primary lymphedema; however, edema may also involve the upper extremities, face, or genitalia (6). Similar findings were noted in this patient.

Differentially we thought parasitic infestation, infection, deep vein thrombosis, secondary to tumors, nodal resection and trauma. In our case filariasis was excluded by doing CFT for filaria, Doppler ultrasonography of affected limbs and abdominal ultrasonography was done and no evidence of deep vein thrombosis and obstructive lesions was found. This patient had no history of trauma and no history of surgical procedure. Radionuclide lymphangioscintigraphy is generally considered the gold standard for the diagnosis of lymphedema. The procedure is minimally invasive, repeatable, easy to perform, and harmless to lymphatic endothelium. Patients with primary lymphedema may have delayed or absent tracer transport, lack or paucity of lymphatic channels, retrograde diffusion (backflow) and poorly visualized or absent regional lymph nodes on lynphoscintigraphy.
Several author found absent or delayed tracer transport in primary lymphedema by isotope lymphangioscintigrphy (7). Lymphoscintigraphy using Tc- 99m nanocolloid was done and non-visualized lymphatic channel and lymph nodes in right lower limb with no ascent of tracer into the lymphatic vessels of the right lower extremity was found in early and delayed images. Gates also reported a case of idiopathic primary congenital lymphedema and he found aplasia of lymphatic channel in isotopic lymphoscintigraphy (8). The management of primary lymphedema is usually conservative and successful for most patients. When medical management fails, surgical intervention is indicated as the last option.

\section{CONCLUSION}

Idiopathic congenital lymphedema is a rare disease in children. Diagnosis of congenital lymphedema is made from a carefully taken clinical history including family history and findings in the physical examination. Physical examination findings can be quite helpful in distinguishing lymphedema from other causes of swollen extremities. In cases where the diagnosis is unclear, radionuclide lymphangioscintigrphy should be performed to confirm the presence or absence of lymphatic dysfunction. In most cases the management of primary lymphedema is conservative but in patients who fail to respond to conservative management, surgical intervention may be suggested.

\section{RERERENCES}

1. Smeltzer DM, Stickler GB, Schirger A. Primary lymphedema in children and adolescents: a follow-up study and review. Pediatrics. 1985;76:206-18

2. Wright NB, Carty HM. The swollen leg andprimary lymphedema. Arch Dis Child. 1994; 71:44-49

3. Fonkalsrud EW, CoulsonWF. Management of congenital lymphedema in infant and children. Ann Surg.1973;177:280-85

4. Shinawi M. Lymphedema of the Lower Extremity:Is It Genetic or Nongenetic? Clinical Pediatrics. 2007; 46(9): 835-41

5. Wananukul S, Jittitaworn S. Primary congenital lymphedema involving limbs and genitalia. J Med Assoc Thai. 2005; 88 (12): 1958-61

6. Sim YJ, Seo JH. Congenital lymphedema of the upper extremity. Eur J Phys Rehabil Med. 2008; 44: 89-91

7. Eric WF, Walter FC. Management of Congenital Lymphedema in Infants and Children. Ann. Surg. 1973; 77(3): 280-85

8. Gates G F, Dore EK. Primary congenital lymphedema in infancy evaluated by isotope lymphoscintigraphy. Journal of Nuclear Med.1971; 12(6): 315-17 